\title{
Anne Gotman, Ce que la religion fait aux gens. Sociologie des croyances intimes
}

Paris, Éditions de la Maison des sciences de l'homme, 2013, 292 p.

Pierre Lassave

\section{(Q) OpenEdition}

\section{Journals}

Édition électronique

URL : http://journals.openedition.org/assr/25489

DOI : $10.4000 /$ assr. 25489

ISSN : $1777-5825$

Éditeur

Éditions de l'EHESS

Édition imprimée

Date de publication : 30 décembre 2013

Pagination : 203

ISSN : 0335-5985

Référence électronique

Pierre Lassave, "Anne Gotman, Ce que la religion fait aux gens. Sociologie des croyances intimes », Archives de sciences sociales des religions [En ligne], 164 | 2013, mis en ligne le 21 février 2014, consulté le 21 septembre 2020. URL : http://journals.openedition.org/assr/25489 ; DOI : https://doi.org/ $10.4000 /$ assr.25489

Ce document a été généré automatiquement le 21 septembre 2020.

(c) Archives de sciences sociales des religions 


\section{Anne Gotman, Ce que la religion fait aux gens. Sociologie des croyances intimes}

Paris, Éditions de la Maison des sciences de l'homme, 2013, 292 p.

\section{Pierre Lassave}

\section{RÉFÉRENCE}

Anne Gotman, Ce que la religion fait aux gens. Sociologie des croyances intimes, Paris, Éditions de la Maison des sciences de l'homme, 2013 
1 Quelle place occupe encore la religion dans la vie quotidienne des fidèles? À quel genre d'activités donne-t-elle lieu et selon quelles modalités? De quelle manière ces activités interviennent-elles dans les différents moments de la vie? Jusqu'à quel point engagent-elles les individus? C'est à ces questions que tente de répondre cet essai à partir d'une enquête approfondie auprès d'une dizaine de personnes qui livrent dans le détail la nature et les formes de leur vie religieuse. Des Français contemporains, catholiques et juifs essentiellement, racontent ainsi par le menu leur vie spirituelle et cultuelle avec ses hauts et ses bas. Nous sommes loin de la comptabilité des appartenances déclarées ou des sondages à large échelle sur l'évolution des croyances.

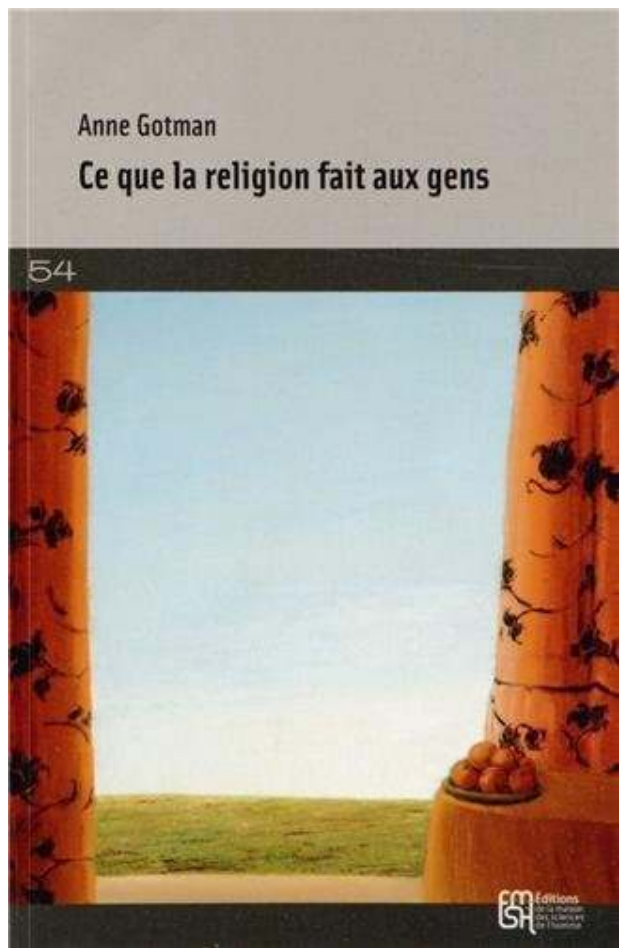

2 Sociologue des modes d'habiter, de la transmission patrimoniale et des formes de l'hospitalité, Anne Gotman est connue pour la finesse de ses analyses d'entretiens en profondeur qui révèlent l'enchevêtrement de processus mentaux et d'institutions sociales majeures. Elle applique ici sa «méthode casuistique » (partir d'études de cas individuels sur ce que disent les gens de la religion à laquelle ils déclarent souscrire) aux idées, sentiments, souvenirs voire anecdotes qui composent le discours sollicité de croyants ordinaires. Plutôt que de partir d'une définition préconçue de ce qu'est la religion, l'auteur s'intéresse à ce que la référence religieuse induit en termes de pratiques quotidiennes et de représentations du monde. Dans les pas de William James, il s'agit de reconnaître l'arbre à ses fruits.

3 La préface aux allures de confession personnelle donne d'emblée les raisons de ce nouveau terrain d'enquête. Née dans une famille juive de l'Est décimée par les déportations et la Shoah, la collégienne au prénom bien français et au patronyme germanique a d'abord pris son empreinte culturelle sous l'angle objectif du drame historique. Bien que non pratiquants, ses parents ne lui ont pas moins donné à assister aux fêtes rituelles chez des amis, à apprendre l'hébreu et à entrer dans une synagogue. Éduquée à l'école publique, entraînée au raisonnement cartésien et rompue aux valeurs humanistes, ce n'est qu'à l'université que le poids de l'héritage judaïque a dévoilé pour elle sa dimension universelle. Mais ce n'est que tardivement, au moment d'ailleurs où la religion préoccupe l'espace public, que la sociologue se retourne sur la dimension religieuse de son propre héritage et en fait un objet d'enquête.

Elle revisite pour cela ce que sa discipline universitaire dit de la religion en ses définitions extensives (diffusion du sacré) ou restrictives (croyance en des êtres surnaturels), substantives (nature irréductible du religieux) ou fonctionnelles (ce que la religion fait socialement parlant). Mais il semble que la philosophie soit plus utile à la définition de son approche. Avec Jacques Bouveresse, elle constate par exemple l'écart entre sa religion plus ou moins faite et les doctrines et traditions célébrées; avec 
Jürgen Habermas, elle s'assure que les fidèles peuvent aujourd'hui parler de leur religion dans le langage commun des bonnes raisons ; avec Ludwig Wittgenstein, elle ne préjuge de la religion qu'aux airs de parenté entre les innombrables formes de vie à laquelle elle donne lieu.

Il serait fastidieux de résumer ici les portraits successifs des interlocuteurs mis à contribution. Cela risquerait d'ailleurs de gâcher le plaisir de lire une analyse de contenu dont la pertinence sociologique s'associe heureusement à une rare qualité littéraire. Évoquons seulement cette fidèle assidue depuis la petite enfance, cadre d'entreprise passée à la retraite et de plus en plus engagée dans la vie de l'Église quelles que soient ses réserves sur certains points de doctrine dépassés et surtout en dépit pour elle d'un dieu trop souvent muet et absent. Lui fait écho à fronts renversés cet autre jeune retraité qui s'ennuyait enfant à la messe mais dont les exigences morales de la paternité, puis un divorce et une nouvelle union n'ont cessé de provoquer en lui le dialogue intime avec le divin. À l'instar d'un autre parcours, celui d'un chef d'entreprise qui réinvestit son savoir-faire dans l'émancipation de ses proches, les " exercices spirituels " d'Ignace de Loyola s'avèrent encore très utiles à l'approche volontaire de ce qui dépasse l'entendement et fait entrevoir la paix de l'âme. Les mêmes péripéties de la foi se retrouvent dans les récits marqués au sceau du judaïsme avec toutefois une mention spéciale pour l'ancrage des gestes et des sentiments dans le foyer familial, véritable base matérielle de l'observance. Souvent proches du premier cas évoqué dans l'écart qui subsiste entre le divin et la religion, ces récits s'en distinguent cependant par la dimension collective de la pratique. Les petits arrangements avec la règle occupent une grande part des dépenses d'énergie pour prolonger l'héritage reçu au gré des aléas de l'histoire. Les situations de mariage entre conjoints de religions différentes sont à cet égard éclairantes. On suit ainsi les interrogations d'un couple qui se forme en Israël entre une jeune femme belge qui s'est rendue quelques années à Jérusalem pour « devenir juive sans y penser » et un étudiant allemand en théologie protestante, connaissant mieux qu'elle l'hébreu et le Talmud. Une fois de plus l'éducation à donner aux enfants suscite des négociations serrées : juifs par la mère, ils seront éduqués dans des institutions juives libérales tout en participant également aux fêtes chrétiennes de leur pays, la France, fêtes observées par les parents de la branche paternelle. L'analyste note avec son interlocutrice que cette alliance a été rendue possible et durable parce que le mari n'est pas devenu pasteur mais historien des religions. Le mariage des dieux impose des limites à ne pas franchir.

Dans la complexité des situations relatées et des raisons invoquées par les acteurs, le lecteur se surprend parfois à se demander quel est au juste le sujet de l'énoncé. Le style indirect libre qu'emploie Anne Gotman pour nous faire comprendre les choses de l'intérieur exige d'ailleurs parfois quelque mise au point en note de bas de page pour savoir qui parle (p.162). L'énoncé chemine pourtant dans un balancement équilibré entre les inférences générales et les illustrations factuelles. Mais parfois une certaine symbiose semble s'installer entre le récit recueilli et celui de l'analyste. C'est un peu le cas à propos de cette femme devenue rabbin en suivant un parcours de formation et d'expérience relativement linéaire malgré la nouveauté historique de sa condition. Le judaïsme n'existant que des multiples obligations qu'il exige, tout ce parcours est animé par le service rendu à cette fin à la communauté. La préférence donnée par l'interlocutrice aux actes par rapport aux discours débouche ainsi côté analyse sur une 
sorte d'éloge d'une forme de vie consistant à mieux penser ses actes en les accomplissant.

7 Cette traversée des formes ordinaires de la vie religieuse dans la société européenne contemporaine ne s'appuie pas moins sur de solides références théoriques, telles par exemple les réflexions de Georg Simmel sur la transfiguration religieuse d'activités profanes associées par la tradition. Mais plutôt que d'inférer de ses récits situés, choisis et limités, des traits trop généraux sur le devenir religieux à l'âge séculier, l'auteur construit soigneusement son objet à l'écart des considérations macrosociologiques sur les conflits et les compromis entre modernité et religions. Elle ne se contente pas de faire état de la variété des formes d'appropriation d'héritages plus ou moins prégnants selon les histoires personnelles mais insiste sur l'indissociation des croyances et des pratiques. Au point de remplacer l'usage savant de ces deux derniers mots par la notion d'activité qui les réunit. Celle-ci "permet d'appréhender ce qui agit dans la transmission de la religion, la manière dont les gens la font, l'état d'esprit dans lequel ils y consacrent du temps et y font vivre leurs convictions » (p. 269). Autant d'activités ordinaires aussi, telles celles de chanter, de danser, de boire ou de lire qui n'ont rien de religieux en elles-mêmes, mais dont l'alchimie particulière qu'opère entre elles une signification transcendante fabrique une religion ainsi que Simmel l'avait déjà montré. Une particularité qui doit beaucoup à l'intention continuée comme l'avait également noté James réfléchissant sur la "volonté de croire». Grâce à elle en effet les contemporains des temps séculiers continuent à s'affranchir des contraintes et des limites de la simple raison lorsque la situation de peine ou d'enthousiasme impose de bouleverser ses cadres de pensée et de vie.

Prolongeant à sa manière de trop rares études de cas qualitatives sur les formes banales de l'activité religieuse dans nos sociétés, comme celle de Robert Wuthnow aux ÉtatsUnis (Growing up Religious. Christians and Jews and their Journeys of Faith, 1999), cette enquête exploratoire réussit à convaincre de ce que l'approche compréhensive peut apporter à la connaissance de processus intimes pour le moins complexes. Loin donc des «métanarrations universalisantes » sur la modernité religieuse et loin également de la comptabilité nécessaire mais forcément réductrice des pratiques et des opinions. On ne peut que souhaiter que cet essai réussi et fort bien écrit suscite des vocations pour porter un regard attentif à d'autres traditions religieuses (on pense tout particulièrement à l'islam en France dont la connaissance ordinaire est noyée sous les clichés) et à d'autres sociétés (dans un esprit comparatif). 